\title{
EL ARYBALLOS CORINTIO DE LA NECRÓPOLIS DE MILMANDA (VIMBODÍ, TARRAGONA) Y SU CRONOLOGÍA
}

\author{
POR \\ RAIMON GRAELLS I FABREGAT \\ Universitat de Lleida, Departament d'Història, Secció d'Arqueologia, Prehistòria i Història antiga.
}

\begin{abstract}
RESUMEN
Se presenta una revisión sobre el aryballos de la necrópolis de Milmanda, prestando especial atención a su cronología. Para este estudio es conveniente redefinir los criterios de atribución tipológica, forma y decoración, así como considerar de manera sumaria otros aryballoi de s.VI a.C. encontrados en Cataluña.
\end{abstract}

\section{SUMMARY}

A revision is presented on the aryballos of the necropolis of Milmanda, paying special attention to its chronology. For this study it is convenient to redefine the approaches of typological attribution, its forms and decoration, as well as to consider in a brief way other aryballoi of VI century BC found in Catalonia.

PALABRAS CLAVE: Aryballos, Milmanda, tipología, Cataluña, s. VI a.C.

KEY WORDS: Aryballos, Milmanda, typology, Catalonia, sixth century B.C.

En el marco de un estudio sobre la necrópolis de Milmanda, se consideró presentar de forma individual algunos de sus materiales más significativos. Éste es el caso que nos ocupa ahora, presentando una relectura del tipo, la decoración y la cronología del aryballos ${ }^{1}$. Esta nueva propuesta supone algunos cambios para la cronología de elementos que han encontrado paralelos en la necrópolis de Milmanda y han basado su datación, sin crítica, en la ofrecida por la primera publicación del aryballos (Ramón, 1989). Aún así, cabe destacar que éste aryballos continua siendo la importación griega más antigua documentada en el interior de Cataluña junto al lekythos del Puig Cardener (Cabrera, 2000: 171).

\footnotetext{
1 Agradezco a los Dres. Paloma Cabrera, Nuria Rafel y Joaquin Ruiz de Arbulo sus comentarios y la revisión del texto en sus distintas fases.
}

La necrópolis de Milmanda (Vimbodí, Conca de Barberà, Tarragona), se encuentra en una ladera de una pequeña sierra en medio de la amplia vía de paso del curso alto del río Francolí, en la parte más al norte de la provincia de Tarragona y cerca del límite entre la comarca de la Conca de Barberà y Les Garrigues que comunica la costa tarraconense con el llano leridano.

La necrópolis se descubrió por unos trabajos agrícolas y fué totalmente excavada por el aficionado local sr. R. Vidal a partir del año 1975. El estudio de los materiales y de los pocos conjuntos que se han podido identificar sobre las notas y fotos de la excavación ${ }^{2}$, permite encuadrar esta necrópolis en un lapso de tiempo que abarca desde finales del s.VII hasta mediados de s.VI a.C.

El aryballos se ha publicado en dos ocasiones por E. Ramón, en 1989 y en 1995. Fué tratado de manera monográfica en una primera publicación dada la innegable importancia que supone la presencia de este elemento importado en el interior de Cataluña. Posteriormente, volvió a publicarlo en un trabajo que sintetizaba las características y algunos elementos significativos de la necrópolis de Milmanda. En esta publicación, la autora identificó la pieza dentro del tipo A de Payne (Ramón, 1995: 109), basándose principalmente en la decoración central del cuerpo (un cuadrifolio, una estrella de cinco puntas y una decoración a cuadros de ajedrez en el frontal del asa). Tipo y decoración con paralelos en el grupo B de las tumbas de Rhitsona (Ramón, 1995: 110), que se incluyen en el Corintio Medio, con una datación entre 595/590-575 a.C.. La característica para considerar este ejemplar dentro del tipo A de Payne no era la decoración, sinó la forma, especialmente el asa y la boca, que como veremos se diferencian de manera clara de los tipos B y más sutilmente del C.

\footnotetext{
2 Los materiales se encuentran divididos entre el Museu Comarcal de la Conca de Barberà, en Montblanc, y el Museu de la Vida Rural de l'Espluga de Francolí. Las fotos se encuentran en el archivo del MCCB. Agradezco desde aquí al sr. Maties Soler y la srta. Dolors Mestres del MCCB, por su disposición y amabilidad durante mis visitas a su institución.
} 
El aryballos apareció asociado a una de las pocas tumbas de las que se dispone de información. El conjunto se componía por una urna de orejetas a mano y algunas armas (espada, punta de lanza y regatón).

En Cataluña, se conocen otros aryballoi. Una visión general nos ayudará a comprender y a concretar el momento de su aparición y la dinámica de su presencia en el nordeste peninsular. De norte a sur de Cataluña encontramos un ejemplar en la Palaiápolis d'Empórion (St.Martí d'Empúries), identificado en el cuadro 9000, y corresponde a un borde de aryballos globular (Aquilué et alii, 2000: 308; fig.21, 4, fase IIIc), con la pintura muy perdida, relacionable con otros objetos del Corintio Reciente de Ampurias. Se fecha en la fase IIIc de la intervención arqueológica, entre el 540 y el 500 a.C. (Aquilué et alii, 2000: 308, Fig.21-4 fase IIIc).

De las distintas necrópolis de Ampurias provienen importantes lotes formados por piezas de diversas procedencias. El primero de ellos se encontró en la inhumación 57 de la Necrópolis Bonjoan, situado a la izquierda del difunto. De pasta amarillenta, y unas dimensiones de alt $55 \mathrm{~mm}$ y diám boca: $35 \mathrm{~mm}$, con decoración acanalada en el cuerpo. Se fecha a finales de s.VI e inicios del V a.C., de producción naucrática (Almagro, 1953: 187-198, fig.168; Trías, 1967: 42, lam.VI.2) ${ }^{3}$.

Dos aryballoi más en la incineración 4 de la necrópolis de la Muralla N.E., de tipo corintio pero indeterminados a causa de su estado de conservación, se fechan entre el 530-500 a.C. por su asociación a lekythoi áticos (Almagro-Basch, 1955; Trías, 1967: 81). En la misma necrópolis, se documenta otro aryballos en la inhumación 2, con el cuerpo globular (h: $63 \mathrm{~mm}$ y $44 \mathrm{~mm}$ de diám boca), que se fecha a partir de los numerosos paralelos en la necrópolis de Rithsona entre el 550 y el 500 a.C. (Trías, 1967: 33, lam.I.7) ${ }^{4}$.

En la tumba 4 de la necrópolis Mateu ${ }^{5}$, apareció un ejemplar del tipo B-1 de Payne, con el cuerpo globular, asa plana vertical, boca discoidal, alt $53 \mathrm{~mm}$ y $38 \mathrm{~mm}$ diám boca., recubierto por un engobe amarillo y decoración muy perdida. Su posición en la tumba resulta interesante al situarse en la mano derecha del difunto ${ }^{6}$. La cronología propuesta, entorno al 570 a.C. (AlmagroBasch, 1953: 228, fig. 191; Trías, 1967: 31-32, lam.I.2), a priori parece quizás un poco alta si nos basamos en las necrópolis en uso en ese momento.

\footnotetext{
3 Inventariado como Trías-32.

4 Inventariado como Trías-6.

5 Inventariado como Trías.-2

6 Recordemos que esta forma de enterramiento con el aryballos en la mano tiene un simbolismo innegable en el ritual funerario griego, que se identifica con el estatus de ciudadano. V. por ej. la "tumba del Atleta" de Taranto, con un alabastron.
}

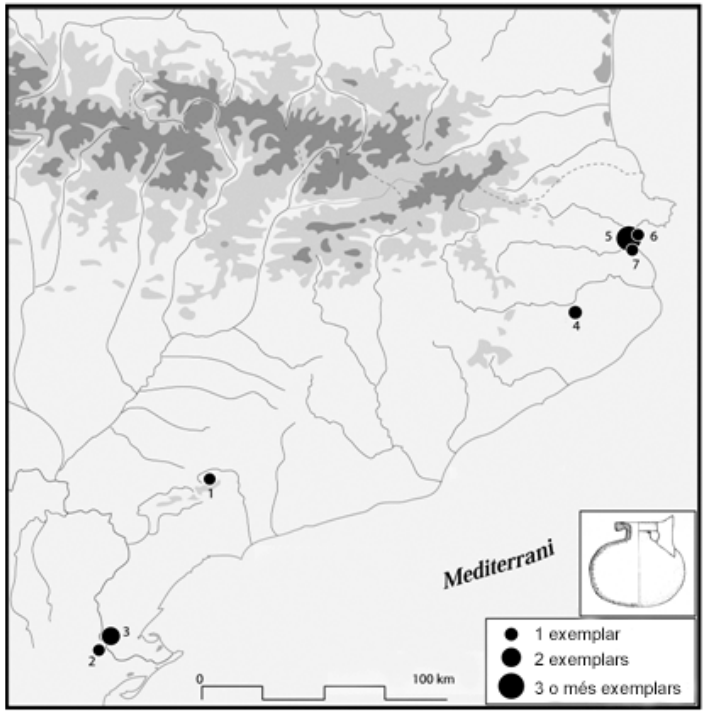

Figura 1. Mapa de distribución de los aryballoi y oil bottles en Cataluña: 1. necròpolis de Milmanda (Vimbodí); 2. necrópolis de Mianes (Sta. Bàrbara); 3. necròpolis de Mas de Mussols (La Palma); 4. Mas Gusó (Bellcaire d'Empordà);

5. Empúries (necrópolis i hábitat, l'Escala); 6. Palaiàpolis d'Empúries (Sant Martí d'Empúries); 7. necrópolis de Vilanera (1'Escala).

Cerca de Empúries, en el yacimiento de Mas Gusó (Bellcaire d'Empordà), se identificó un fragmento de aryballos corintio globular de tipo B de Payne, fechado en el Corintio Medio, a mediados del s.VI a.C. (Casas i Soler, 2000: 355, Fig. 7, 5).

No podemos evitar extrañarnos por la curiosa ausencia de aryballoi en la zona central de la costa catalana, que goza de una intensa actividad arqueológica además de la tradición investigadora, que ha documentado diversos materiales griegos de mediados de s.VI a.C. en el Vallès ${ }^{7}$ o el Penedès ${ }^{8}$. Seguramente este tipo de materiales estén aún por aparecer.

En la desembocadura del Ebro, se encuentran de nuevo otros hallazgos de aryballoi. En la tumba 4 de la necrópolis de Mianes (Santa Bàrbara), se supone la presencia de un aryballos a partir del hallazgo de un fragmento de borde plano y pintado con dos líneas paralelas (Maluquer, 1987: 125). En cambio en la necrópolis de Mas de Mussols (La Palma) se encontraron, durante las excavaciones de urgencia de F. Esteve Gálvez, dos aryballoi globulares

\footnotetext{
7 Un oenochoe de cerámica gris en la tumba del Guerrero de la Granja de Soley en Sta. Perpètua de la Mogoda (Sanmartí et alii, 1982).

8 Distintos tipos en la Ciutadella d'Alorda Park en Calafell o en el campo de silos de Avinyonet del Penedès.
} 
(Maluquer, 19894b: fig.11-12), uno de producción corintia y el otro de imitación local ${ }^{9}$. Según indicaciones del descubridor (Villalbí, 1999: 74), esos ejemplares, aparecieron junto a una urna de orejetas, un anillo de plata, un broche de cinturón de tres garfios y una oil bottle en una misma tumba. Cabe resaltar que Maluquer en su monografía sobre sus excavaciones en el Mas de Mussols (1984b) publica esta tumba como un hallazgo anterior a sus excavaciones sin citar que correspondía al salvamento llevado a cabo por Esteve y sus colaboradores. Así hoy en día se conoce la tumba como T.X a partir de la obra de Maluquer, mientras para Esteve corresponde a la S.18 (Esteve, 1999: 61, n.2). Esta combinación es súmamente relevante ya que plantea el problema sobre el comercio de distintas producciones tanto fenicias como griegas en las bocas del Ebro. La citada oil bottle corresponde a la forma 3 de Bisi (1970), más concretamente al tipo 2361 de Ramón (1982), que encuentra la mayoría de sus paralelos en Cerdeña ${ }^{10}$. Más próximos, encontramos paralelos en la necrópolis de Vilanera (Agustí et alii, 2002: 81), del Puig des Molins ${ }^{11}$. Ampollas de tipos similares, aparecen también en la costa del levante peninsular en la fase III y en la VI de la Fonteta (González-Prats, Ruiz y García, 1999: Fig.14; González-Prats, 1999: Fig.5). El tipo correspondiente a Mas de Mussols, ha sido propuesto como una producción de talleres sardos (Ramón, 1982: 32), opinión basada en la abundancia de este tipo (2361) y de tipos similares (1351, 1361, 2351) en las necrópolis de Bithia y Mozia. Esta producción se vehicularía hacia Mas de Mussols desde Ibiza (Ramón, 1982: 37). La cronología varía de s.VII hasta mitad del s.VI a.C., con perfiles que van desde la esfericidad a formas más alargadas; de presentar pies anulares a encontralos sin pie y con variaciones en las secciones de las asas. Los cuellos, de distinto grosor, siempre aparecen con estrangulamientos. El ejemplar de Mas de Mussols ha sido fechado entre el 600 y el 550 a.C. (Ramón, 1982: 26;

\footnotetext{
9 Imitaciones de aryballoi no se documentan en la Península aparte de este ejemplar, pero sí alguna pieza inspirada en ellos, como la granada de la tumba 4 de la necrópolis de la Loma del Peinado en Casillas de Martos (Jaén), interpretada por Maluquer como un aryballos (Maluquer, 1984a: 20, fig.7).

${ }^{10}$ Mozia (Ramón, 1982: cat.22), Necrópolis de Nora (18911892) (ibidem: cat.28), T.17-1955 y T.?-1933 de la necrópolis de Bithia, también aprovechamos esta nota par incluir un ejemplar de Cartago (ibidem: cat.4).

${ }^{11}$ Cat.56 (Ramón, 1982); t. 29 Via Romana-Can Partit (Gómez-Bellard, 1990: 111-112, fig.102). A pesar que la presencia de oil bottles se constata en la Península Ibérica, con tipos distintos de Ramón en la cata A de la Peña Negra (González-Prats, 1979a: 194, fig.174.1317), Sa Caleta (Ramón, 1999: 165-166 y 184, fig.6 XIII-8 y fig. 17 p-57 y d-2) Puig d'es Molins (Gómez-Bellard, 2000: fig.7), Chorreras (Belén y Pereira, 1985: 6-7), Cruz del Negro (Belén y Pereira, 1985: fig. 3.4-5), Riotinto, Cerro de la Mora y Saladares
}

Vives, 2005: 140) ${ }^{12}$, fecha que resulta difícil de considerar para los aryballoi de tipo griego, para los que nos resultaría más convincente una datación entre el 575 y el 550 a.C., más acorde con la presencia del broche de cinturón y de la urna de orejetas del mismo hallazgo. Estos dos últimos objetos plantean la duda sobre si el conjunto correspondía o no a una misma tumba, debido a la aparente distancia cronológica entre los aryballoi, la oil bottle y el anillo, que formarían un grupo cronológicamente antiguo (segundo cuarto del s.VI a.C.), y la urna y el broche, claramente de segunda mitad de s.VI a.C. ${ }^{13}$.

Por otro lado, existe un abundante número de aryballoi depositados en el Museu Arqueològic de Catalunya en sus sedes de Barcelona y Girona ${ }^{14}$. Unos son de las primeras excavaciones en Ampurias y otros, pertenecientes a las antiguas colecciones Guerin de Barcelona y Catalina Albert de l'Escala, son, muy probablemente también, de Ampurias (Trias, 1967: 41-42):

1. MAC.-405, Tipo B-1 de Payne, con el cuerpo globular, asa plana vertical, boca discoidal, $71 \mathrm{~mm}$ alt y $45 \mathrm{~mm}$ diam boca. Decoración tardía dentro de la série de aryballoi con el motivo de la sirena, con un paralelo en la t.86 de Rithsona que ofrece una cronología de c. 570 a.C. (Trías, 1967: 31, lam. I.1) ${ }^{15}$. El tipo de decoración con "sirena" ofrece un amplio espectro cronológico que va del EC, por ejemplo en un ejemplar del nivel 9-10 del depósito 1 de Tocra (Boardman y Hayes, 1973: 11, Pl. 2.1845), hasta el MC, con un ejemplo en Rouen, procedente de Pozzuoli y atribuido al pintor de Calaureia (Benson, 1981: 172, Pl. 38.9). Al mismo tiempo las formas pueden variar substancialmente, desde la esfericidad de las piezas comentadas más arriba a los aryballoi con base plana o pie anular ${ }^{16}$.

2. MAC.-322 ${ }^{17}$, Tipo B-1 de Payne, globular con el asa vertical y la boca discoidal, con unas dimensiones de 59 mm H. y 39 mm. D. Boca, y una decoración del llamado tipo "grupo de los guerreros" ${ }^{18}$, con cinco guerreros pintados. Presenta una datación, aplicable al ejemplar de la colección Guerin, de segundo cuarto del s.VI a.C. (Trías,

${ }^{12}$ A pesar que el tipo 2361, se fecha en sentido amplio, según los paralelos, entre el último cuarto del s.VII y el primero del VI a.C. (Ramón, 1982: 25-26).

13 Además, F. Esteve, no cita ni el broche ni la urna en su memória (Esteve, 1999: 61), sí lo hace, en cambio, Maluquer (1984b: 39), quién asocia todas las piezas como un probable conjunto funerario.

${ }^{14}$ Para el inventario que sigue, la sede de Barcelona se identificará como MAC y la de Girona como MAG. MDG corresponde al Museu Diocesà de Girona.

15 Inventariado como Trías-1.

${ }^{16}$ Sobre éstos v. Lawrence, 1959: Pl. 92.28 y 92.32.

17 Inventariado como Trías-4.

${ }^{18}$ Round-Shielded Warriors, Payne: 1244-1249; Ure, 1934: 38-41. 
1967: 32, lam. I.4 y 5) que coincide con Payne (575-550 a.C.) pero no con Ure (590-570 a.C.). El motivo encuentra abundantes paralelos en Rithsona, especialmente en los grupos de tumbas $b$ y $c$ (Ure, 1934: 22), aunque son mayoritarios los ejemplos que presentan 3 y 4 guerreros.

3. MAG.-7, Tipo A de Payne, con el cuerpo globular, asa plana vertical, boca plana, $62 \mathrm{~mm}$ alt., $46 \mathrm{~mm}$ diam. Boca, pasta amarilla-verdosa con decoración. Decoración con un Sixfoil (Ure, 1934: 43). La cronología está en la primera mitad del s.VI a.C. (Trias, 1967: 32, lam. I.3; Ure, 1934) ${ }^{19}$.

4. MDG- $3306^{20}$. Pasta amarillenta $44 \mathrm{~mm}$ alt., posible imitación de un modelo naucrático, fechada entre finales de s.VI y principios s.V a.C.. (Trías, 1967: 42, lam VI.3).

5. Colección Guerin (Barcelona) ${ }^{21}$, Tipo B-1 de Payne, globular con la boca discoidal, $55 \mathrm{~mm}$ alt., 40 $\mathrm{mm}$ diam. boca. Decoración tipo "grupo de los guerreros", en este caso tres, con una cronología, de 575-550 a.C. (Trias, 1967: 32-33, lam. I.6). Encontramos abundantes paralelos con motivos pintados de tres guerreros en distintas tumbas de Rithsona: 4.8 (Ure, 1910: 354), 86.68, 86.73-82, 92.7-9, 95B.37-42, 95C.46-48, 99.1516, 125a.4-6, 125b.3, 125c.3-8, 145.16-26 (Ure, 1934).

6. Trias-31, Tipo Payne B-1, con decoración de retícula incisa, con el cuerpo globular, el asa plana vertical, la boca con reborde levemente cónico, $47 \mathrm{~mm}$ alt., $33 \mathrm{~mm}$ diam. Boca y superfície acabada con vidriado azul-verdoso. Corresponde a una producción naucrática o rodia que se fecha a inicios de s.VI a.C. (Trías, 1967: 41, lam. VI.1).

7. MAC-323, Pasta amarillenta y $55 \mathrm{~mm}$ alt., con decoración con relieves prismáticos. Se fecha entre finales del s.VI e inicios del V a.C. (Trías, 1967, 42, lam. VI.4) ${ }^{22}$. Probablemente de producción naucrática o rodia.

8. Colección Catalina Albert (L'Escala), Cuerpo globular gallonado, boca plana discoidal, $60 \mathrm{~mm}$ alt., $40 \mathrm{~m}$. diam. boca, base plana y lisa. Se fecha entre finales de s.VI e inicios del V a.C. (Trias, 1967: 42, lam. VI.5) ${ }^{23}$.

9. MAC-326, Aryballos plástico que representa un pie con sandalia, con una boca de $27 \mathrm{~mm}$ de diam., de probable producción greco oriental (Trías, 1967: 44-45, lam. X.1) ${ }^{24}$.

10. MAG-16, Cuerpo globular, boca plana en disco, arcilla beige clara, $80 \mathrm{~mm}$. alt., decoración a bandas y líneas radiales alrededor del cuello, producción etruscocorintia con una cronología c.550 a.C. (Trías, 1967: 48, lam. XI.1) ${ }^{25}$.

\footnotetext{
${ }^{19}$ Inventariado como Trías-3.

20 Inventariado como Trías-33.

${ }^{21}$ Inventariado como Trías-5.

22 Inventariado como Trías-34.

23 Inventariado como Trías-35.

24 Inventariado como Trías-43.

25 Inventariado como Trías-52.
}

11. MAC- $326^{26}$, Cuerpo piriforme, con pasta amarillenta. $89 \mathrm{~mm}, 55 \mathrm{~mm}$ de boca y $14 \mathrm{~mm}$ de diámetro de pie. Decorado con líneas paralelas horizontales de distintos grosores menos en la parte superior del cuerpo que presenta una serie radial de líneas. Producción etrusco-corintia. Según Trías se fecha a principios de s.VI aC (1967: 48), datación con la que coincidimos para este ejemplar a pesar de encontrar propuestas que sitúan estas producciones en el tercer cuarto del s.VII a.C. (Benson, 1964: 168, pl. 53.2).

12. MAC- $325^{27}$, Forma de pera o calabaza (Trías, 1967: 48), pasta amarillenta, $80 \mathrm{~mm}$. de altura y $37 \mathrm{de}$ diámetro de boca. Decoración de bandas paralelas horizontales y líneas radiales en el cuello. Igual que los dos ejemplares anteriores es una producción etrusco-corintia que se fecha durante la primera mitad del s.VI a.C.

También se documentan aryballoi griegos durante el s.VI a.C. en contextos de Eivissa, en la necrópolis de Medellín (Jiménez y Ortega, 2004: 85-86; Torres, 1999), en Vilajoiosa (Shefton, 1982: 54), en Huelva (Cabrera, 1988-1989: 50 y 56; Fernández-Jurado, Rufete y García, 1991: 75 y 79, fig. 4 y 5; Rouillard, 1977: 400, lam. XV), en Coria del Río, en Málaga (GranAymerich, 1988: 210-211, fig. 10.9 y 10.10), Adra, en la necrópolis de Villaricos (Cabrera, 1988-1989: 56; Shefton, 1982: 54; Trias, 1967: 436, lam. 192). De los que el ejemplar más próximo es el de Villaricos, ya que presenta una línea ajedrezada bajo los motivos figurados.

El uso de perfumes durante el final de la primera edad del Hierro en Cataluña, a pesar de documentar unos pocos recipientes, no parece ser de uso común ni siquiera entre las élites. Eso puede, por otro lado, subrallar la importanca de su presencia, de carácter sumamente restringido. Seguramente la elección de un olor imposible de copiar, identificaría a sus portadores como miembros de un grupo privilegiado. En este punto es necesario preguntarse sobre si realmente se importa el vaso o si es el contenido, el perfume, el elemento que da valor al vaso. Como ha sido propuesto, es probable que los vasos para contener perfumes se fabricasen en los mismos lugares donde en la Antiguiedad se producían los aceites perfumados. El caso de Corinto, de donde proceden muchos de los aryballoi hallados en la Península, se distinguió por la producción del perfume de Iris (Cavallo, 2004: 248) ${ }^{28}$, hecho que ratificaría esa máxima

\footnotetext{
${ }^{26}$ Corresponde al número de inventario publicado por Trías (1967: 48), que repite el número de inventario del aryballos plástico inventariado aquí como n.7. Inventariado como Trías-53.

27 Inventariado como Trías-53.

${ }^{28}$ Sobre este tema v. G.Donato, M.E.Branca y A.Rallo (1975): Sostanze odoroze nel mondo classico, Venezia; B.Rossignoli (2000): "Corinto Afrodite e il commercio di profumi", Hesperia, 12, 195-198. Referencias citadas en Cavallo 2004: 248, n.84.
} 


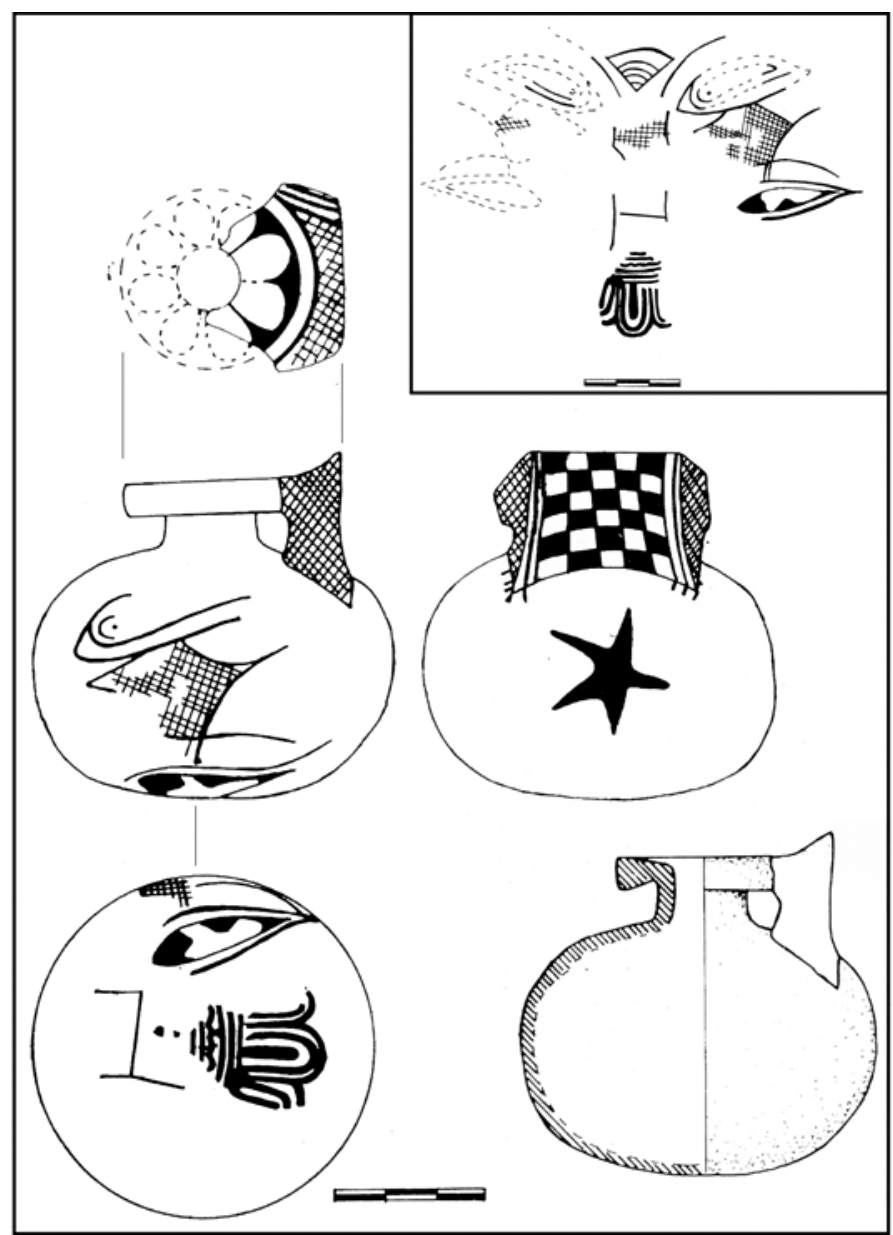

Figura 2. Dibujo del Aryballos con distintas vistas y sección. En el rectángulo superior, desarrollo de la decoración. Según Ramón 1989.

sobre la conjunción de lugar de producción - lugar de embotellamiento - y comercio. De todos modos, cabe la posibilidad de que el contenedor pase a identificarse como símbolo exótico del producto y por lo tanto, merecedor de acompañar en la tumba a sus propietarios, igual que sucede con otras producciones importadas que aparecen de manera recurrente en las tumbas ${ }^{29}$.

La importancia simbólica del perfume tiene sus raíces en el imaginario oriental, que lo considera el sudor o la comida de los dioses, y por lo tanto, símbolo de immortalidad (Olmos, 1985: 14) ${ }^{30}$. En la Península, se

${ }^{29}$ Oenokhóai, copas y ánforas, que combinan su uso como elementos de vajilla del vino con otro uso relacionado con el símbolo del acceso al mismo (con todas sus variables: consumo en vida, consumo después de la muerte, posibilidad de celebrar banquetes, celebración de un banquete funerario, etc). Sobre este problema v. Valenza-Mele (1991: 152, n.5).

${ }^{30}$ Od. 5, 93; 4, 445; Il. 5, 777. ha documentado mayoritáriamente en relación a contextos funerarios, destino privilegiado del perfume si recordamos las protecciones para evitar la corrupción de los cuerpos o el complejo sistema de embalsamaje (Il. XIV, 170; XVI, 680; XXIII, 186-187). El uso de perfumes se documenta tanto en contextos con influencias fenicias como griegas indistintamente. Para los contextos tartésicos, M.Torres (1999) recogió los ejemplos, que se presentan bajo distintas formas de recipientes (alabastra y oil-bottles) en las tumbas 9 y 17 de la necrópolis de la Joya, la tumba 1 del Túmulo 1 de las Cumbres, en el túmulo $\mathrm{H}$ del Acebuchal, en la tumba $\mathrm{B}$ de la necrópolis de Osuna, en la tumba 64 del túmulo 1 de Setefilla, en la tumba de Belvís de Jara y en distintas tumbas de las necrópolis de la Cruz del Negro y Carmona.

Esta costumbre, que encuentra pocos ejemplos durante el s.VI a.C. en Cataluña, verá una importante proliferación de su uso en Ampurias a partir del s.V a.C., como demuestran los abundantes recipientes de pasta vítrea de 
las necrópolis Martí o Bonjoan (Almagro Basch, 1953) ${ }^{31}$, o los alabastra tanto cerámicos como en alabastro (Almagro-Basch, 1953; Trias 1967), especialmente notable con el floruit de la colonia (Fernández y Mezquida, 1997: 52).

Volviendo al ejemplar de Milmanda, se encuentra depositado en el Museu Comarcal de la Conca de Barberà con el número de inventario NM. 1; 22.1. Consiste en unos fragmentos de pasta gris amarillenta de borde, pared y fondo que permiten su reconstrucción. Presenta un cuerpo globular, asa de sección trapezoidal, superfície exterior plana que arranca del borde del disco y termina a un tercio de la altura. Según la ficha, presenta una decoración en barniz negro muy maltrecha, pero después de examinar la pieza, la decoración consiste en pintura negra sobre el fondo blanco (para el que tenemos dudas de si realmente es un engobe o presenta una capa de pintura blanca en toda la superfície). La decoración consiste en una retícula ajedrezada en el asa, ovas en el fondo, roseta de ocho pétalos en la parte superior del disco, y, en la pared del cuerpo una estrella de cinco puntas y un cuadrifólio.

La restauración consistió en juntar los fragmentos así como la limpieza mecánica de la superfície y la reintegración de los espacios vacíos con yeso y aquaplast (Restauración a cargo de A.Ma.León, junio de 1985).

Dimensiones: alt. máxima: $70 \mathrm{~mm}$; diam. cuerpo: 68 mm.; alt.. Asa: 30 mm.; ancho. Asa: 35 mm.; grosor. Asa: $11 \mathrm{~mm}$.

La creación del aryballos de cuerpo esférico privado de base, debe considerarse una invención oriental que encuentra los primeros ejemplares en Egipto en la XVII dinastía (Payne, 1931: 287, n.1) ${ }^{32}$. En ámbito griego, la copia de estas piezas empieza a partir del Protocorintio, con el tipo Round Aryballos A de Payne, caracterizado por una boca en forma de disco plano y una asa gruesa de sección rectangular que forma ángulo recto con la boca y realizados principalmente en fayenza, a modo de imitación de los originales orientales (Payne 1931: 287). En el

${ }^{31}$ T.77 necròpolis Martí (Almagro-Basch, 1953: 81-84); T,23 necròpolis Bonjoan (Almagro-Basch, 1953: 164-166); T.43 necròpolis Bonjoan (Almagro-Basch, 1953: 178-183), T.55 necròpolis Bonjoan (Almagro-Basch, 1953: 193-196); T.57 necròpolis Bonjoan (Almagro-Basch, 1953: 197-198); T.12, necròpolis Granada (Almagro-Basch, 1953: 242-243).

${ }^{32}$ La evolución estilística de esta forma cerámica, según DeCarolis (1991) pero no coincidente con Payne (1931), se puede esquematizar desde las producciones globulares con pequeña asa sobre el hombro (725-700 a.C.. - Protocorintio Arcaico), siguiendo por los tipos llamados piriformes con asa ancha entre el borde y el hombro (700-650 a.C. - Protocorintio Medio I-II - y el 650-590 a.C. - Protocorintio Tardio Transicional), los globulares sin base (640-625 a.C. - Corintio Transicional - y el 625-575 a.C. - Corintio Arcaico), y los globulares con base (590-575 a.C. - Corintio Medio).

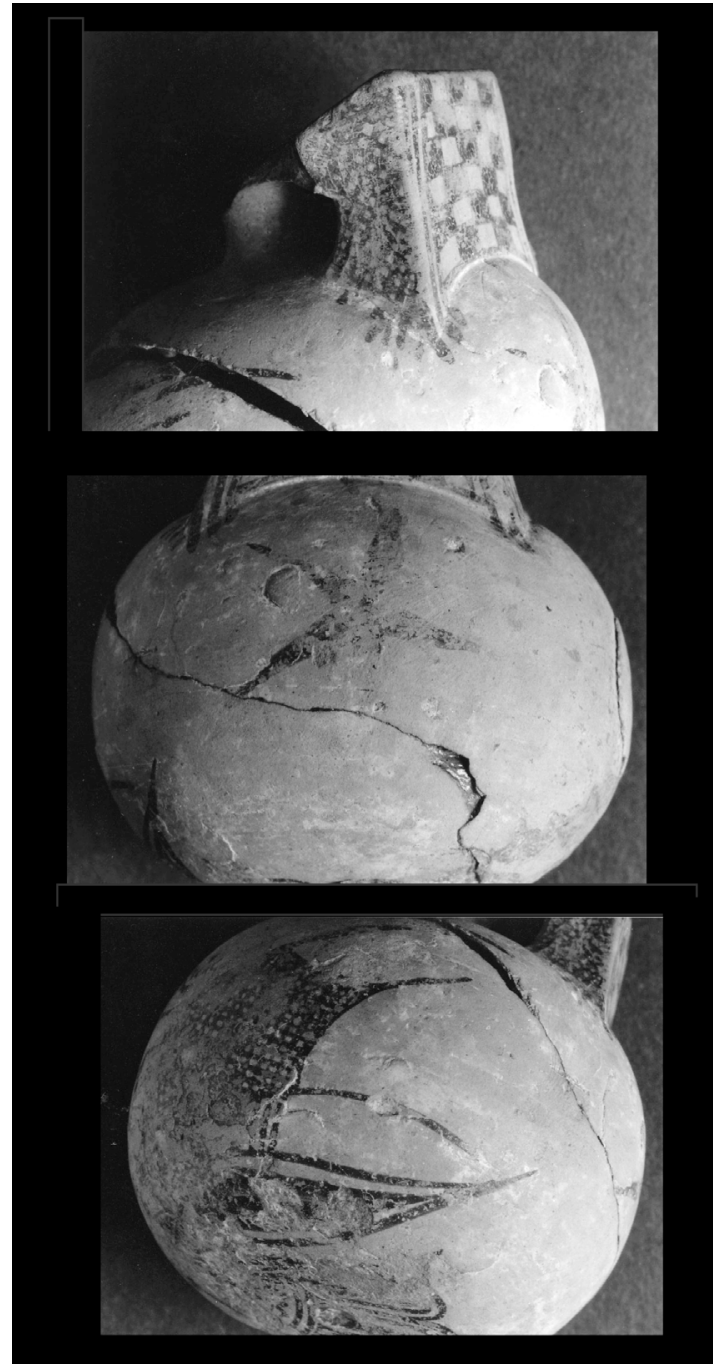

Figura 3. Detalles de la decoración. Arriba, detalle del ajedrezado del asa; en el medio, detalle de la estrella; abajo, detalle de cuadrifóleo. (Fotos E.Ramón y R.Vinyes, MCCB).

Corintio Transicional ${ }^{33}$, se varía la forma, se afianza en el Corintio Arcaico (Payne: 625-575 a.C.; Hopper: 620/615$595 / 590$ a.C.) y se consolida definitivamente en el Corintio Medio (Payne 600-575 a.C.; Hopper: 595/590-575 a.C.). El tipo B de Payne, se caracteriza por una boca en

${ }^{33}$ La cronología sobre este período ha variado desde la propuesta de Payne (1931) que ofrecía un arco entre el 640 y el 625, a partir de la revisión de Hopper (1949) que lo situó definitivamente entre el 630 y el 620/615 a.C. Lo mismo pasa en los otros bloques cronológicos de la cerámica corintia, la cronología de los cuales se añade después de cada uno con las propuestas abraviadas de Payne y Hopper. 


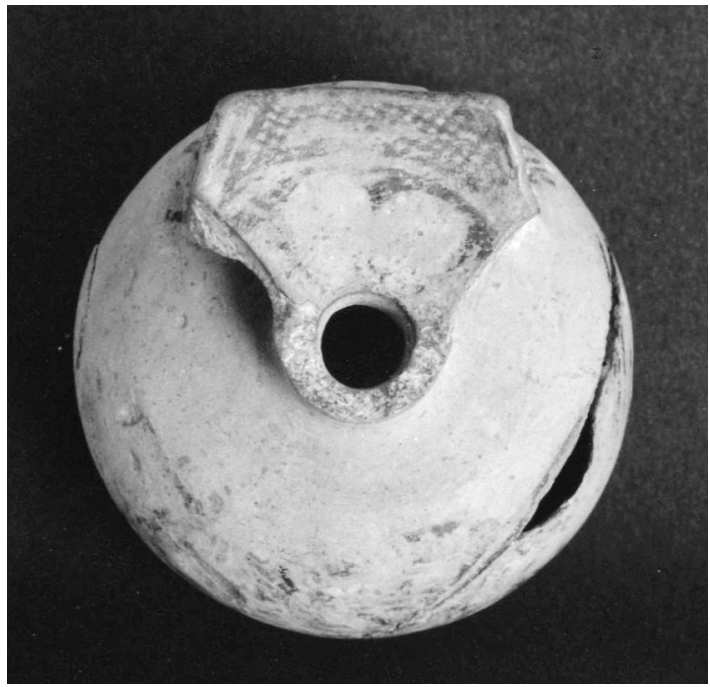

Figura 4. Vista desde arriba, disco y parte superior del asa (Foto E.Ramón y R.Vinyes, MCCB).

forma de disco plano o ligeramente cóncavo y una asa de sección elíptica y silueta en "S" (Payne, 1931: 287). Estas producciones tuvieron una gran cantidad de imitaciones en ámbito etrusco, con la cerámica etrusco-corintia (hasta el 530 a.C. - según DeCarolis, 1991:41-). A partir del Corintio Medio, se abandonan las asas de cinta del tipo B y se recupera el perfil de los aryballoi del tipo A, con asas cúbicas y paredes gruesas. Pero el tipo $\mathrm{C}$, varia la forma de la boca del tipo A, pronunciando, más aún, la concavidad de modo que el borde aparece colgando, simulando exteriormente que se trata de piezas arcaicas de tipo A, pero internamente se realizan con un importante cambio tipológico. El tipo $\mathrm{C}$ tiene unas dimensiones estandarizadas, entre 66 y $74 \mathrm{~mm}$. de altura por un diámetro normal de $68 \mathrm{~mm}$. La decoración característica presenta unos patrones que se repiten en todos los ejemplares de la série:

Decoración del borde del disco, siempre repitiendo un mismo esquema, integrado por dos líneas paralelas en la parte superior e inferior, y en el centro de la franja, una decoración que puede ser o en motivos tipo "S" o en ajedrezado. Este ajedrezado se caracteriza por no tocarse los cuadros negros entre ellos, ya que surgen directamente de las líneas internas (Payne, 1931: Pl.31,5, n.803) ${ }^{34}$.

\footnotetext{
${ }^{34}$ El motivo decorativo del ajedrezado aparece en la cerámica corintia en la fase transicional, usándose para dibujar franjas (Payne, 1931: Pl.12, 4, n.143). A pesar de que no se puede negar que la decoración por oposición de colores de manera geométrica sea una decoración conocida en otros ejemplos, anteriores y cohetáneos tanto en Grecia como en otros puntos del Mediterráneo, siendo uno de los ejemplos más especaculares el mosaico del santuario de la Muela de Cástulo.
}

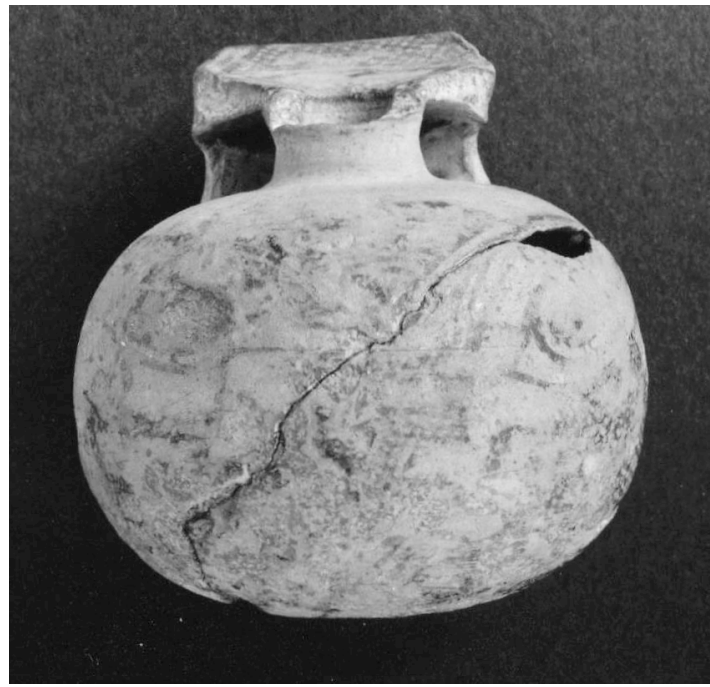

Figura 5. Vista frontal (Foto E.Ramón y R.Vinyes, MCCB).

Decoración de la métopa posterior del asa, donde el dibujo se presenta emmarcado por dos líneas en todos los lados y las decoraciones con ajedrezado se presentan de manera completa, tocándose los cuadros entre sí (Payne, 1931: Pl.31,4, n.806). En los ejemplares en que la métopa posterior presenta otros motivos ${ }^{35}$, esta se emmarca únicamente por los lados, también con motivos de dos líneas paralelas, dejando sin decorar la parte superior e inferior.

Por lo que respecta al ejemplar que tratamos, nos centraremos en los aryballoi decorados con motivos vegetales tipo cuadrifólios ${ }^{36}$, encontrando numerosos paralelos que presentan decoraciones similares pero como señaló en su momento J.L. Benson (1964: 170) con abundantes variantes, como son las correspondientes al ajedrezado del asa, algunos motivos florales del cuerpo, la estrella y los laterales del disco ${ }^{37}$. Según Payne, la decoración con flor de loto cuadrifolia

\footnotetext{
${ }^{35}$ Normalmente retratos femeninos aunque pueden encontrarse cabezas de caballo, pero nunca más líneas horizontales paralelas, características del tipo B. Los retratos femeninos son característicos del MC (Broneer 1955: pl. 51.1; Roebuck, 1955: 159).

${ }^{36}$ V. Ure, 1934: 44, para la distinción entre ejemplares de cuatro, cinco y seis pétalos.

${ }^{37}$ Entre otros v.: Benson, 1964: 170, pl.55,9; Benson, 1981: 173, pl.38,10; Deppert, 1964: 20-21, Taf.16.1 a 16.12; KunzeGötter, 1965: 18, Taf.12.5 a 12.8 i 12.10; Newhall y Benson, 1984: 92, Plate 22.419; Payne, 1931: n.484, n.485; Lullies, 1972: 31, Taf.10.3; Ure, 1934: T.14, 91, 86, 92, 95, 99, 101, 125 y 145 . Así como también otros CVA correspondientes a Leipzig (1), Heidelberg (1), Karlsruhe (1), Zürich (1), Scheuleer (1), Oxford (2), Paris Biblithèque National (1) o Gela (1).
} 


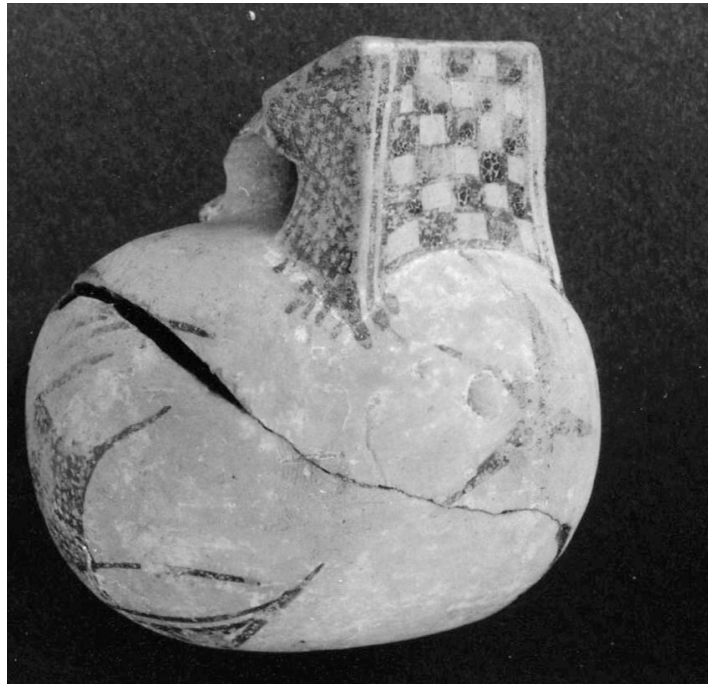

Figura 6. Vista dorsal (Foto E.Ramón y R.Vinyes, MCCB).

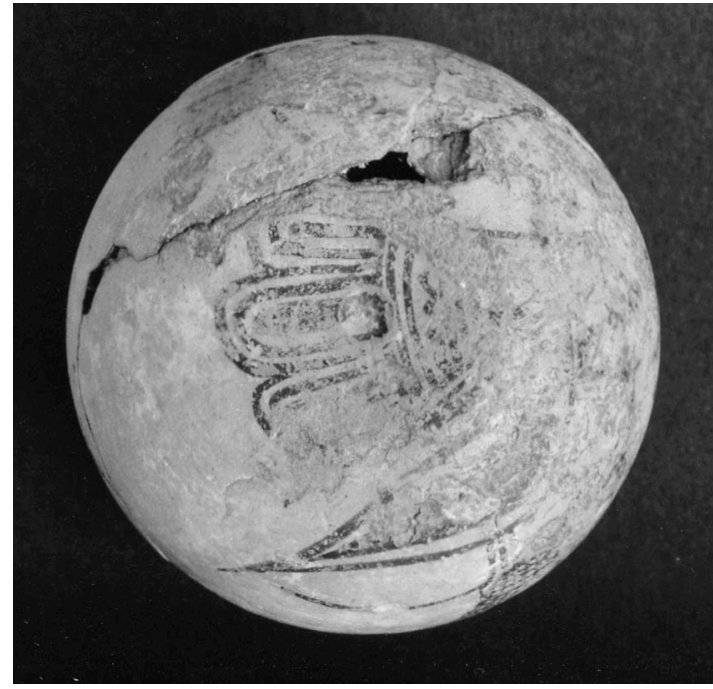

Figura 7. Vista de la base (Foto E.Ramón y R.Vinyes, MCCB).

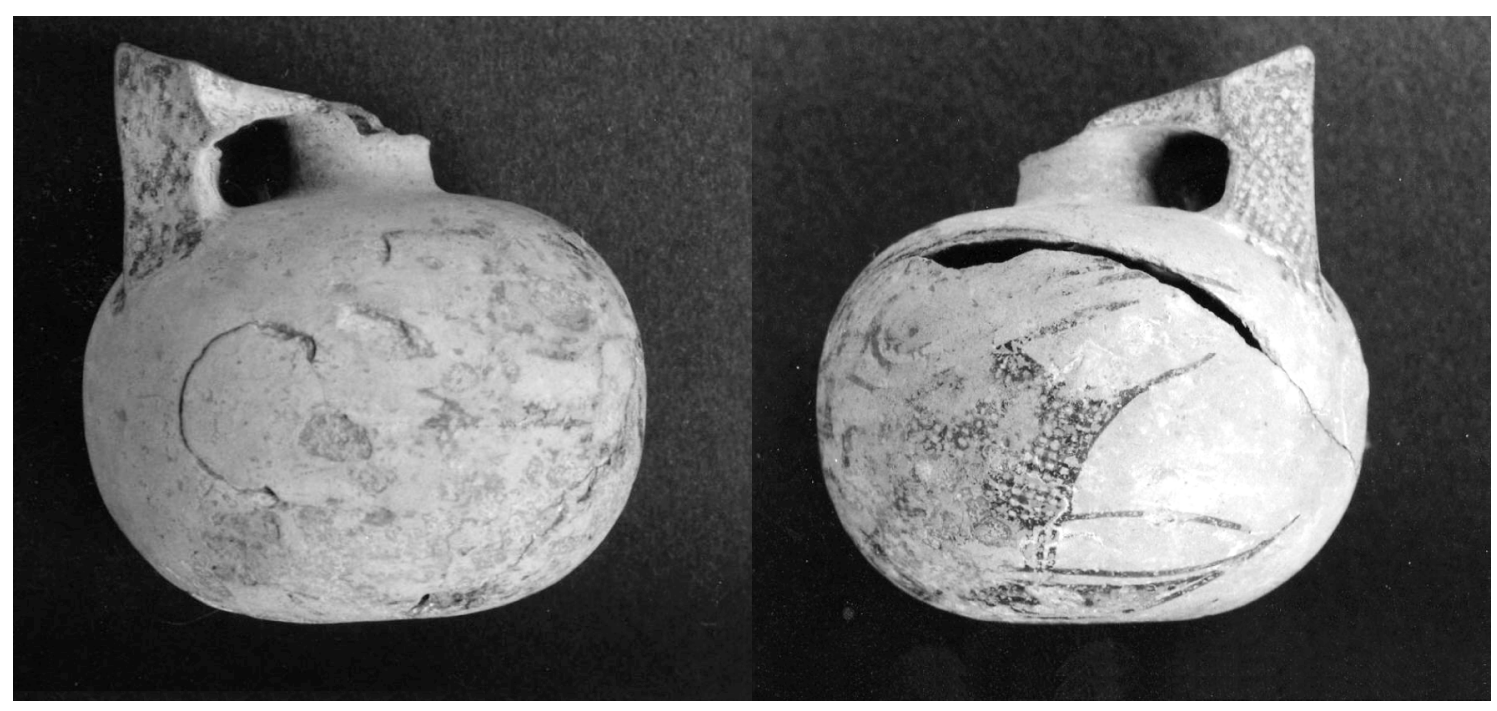

Figura 8. Vistas laterales (Fotos E.Ramón y R.Vinyes, MCCB).

comienza como copia de motivos asirios y durante el Early Corinthian Period irán variando, dando cada vez mayor importancia a la parte central. A partir del Late Corinthian Period es cuando goza de mayor difusión este motivo decorativo (Ure, 1934: 43), igual que el ejemplar de Milmanda, identificado como Payne-D (Payne, 1931: 147: fig.54), que se fecha principalmente en el segundo cuarto del s.VI a.C. (Payne 1931: 148). La estrella de cinco puntas ayuda a situar la pieza en el segundo cuarto del s.VI a.C., ya que el motivo aparece como un elemento decorativo típico del Corintio Tardio, entre el 570 y el 550 a.C. (DeCarolis, 1991).
El tipo al que debe adscribirse el aryballos de Milmanda se identifica como tardío dentro de las séries de aryballoi globulares (Boardman y Hayes, 1973: 7), recibiendo el nombre de Vierblatt-Aryballoi o Quatrefoil Aryballoi (Ure, 1934: 34), que se fecha según E. Kunze-Götte (1965: 18) y también P. Lawrence, en base a la tumba Korakou de Corinto (1964: 103), entre el 575 y el 550 a.C., en cambio, según R.Lullies (1972: 31) alrededor del 550 a.C., opinión reafirmada por el ejemplar de la t.3 de Cozzo Matrice (Cilia, 1980-1981: 617, fig.2).

De esta manera el recipiente de Milmanda pasa de una cronología de 595-570 a.C. (Ramón, 1995), matiza- 


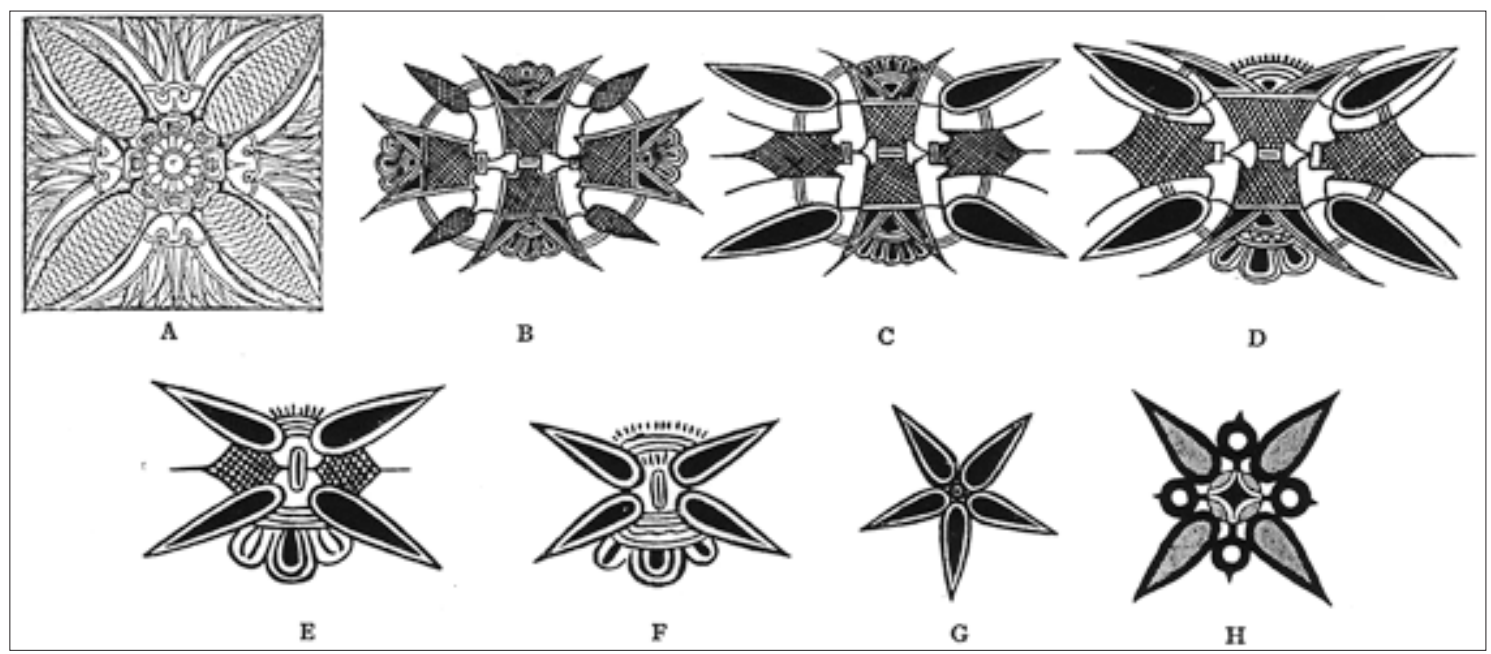

Figura 9. Evolución de las decoraciones de tipo flor de lotus Quatrefoil, según Payne 1931: 147, fig.54.

da por P. Cabrera al situarlo entorno al 570 a.C. (Cabrera, 1996: 46; Cabrera, 2000: 171), a otra datación de 570-550 a.C. que entra en una lógica "comercial" más amplia tanto por el número de elementos importados como por el número de yacimientos con importaciones.

\section{BIBLIOGRAFÍA:}

AGUSTÍ, B., CODINA, D., DEHESA, R., LLINÀS, J., MERINO, J., MONTALBÁN, C., VARGAS, A. (2002): "La necròpolis d'incineració de Vilanera", Sisenes Jornades d'Arqueologia de les comarques gironines, 77-86.

ALMAGRO-BASCH, M. (1953): Las necrópolis de Ampurias, Introducción y necrópolis griegas, Monografías Ampuritanas, III, Barcelona, 1955

ALMAGRO-BASCH, M. (1955): Las necrópolis de Ampurias, necrópolis romanas y necrópolis indígenas, Monografías Ampuritanas, III, Barcelona, 1955.

AQUILUÉ, X., CASTANYER, P., SANTOS, M., TREMOLEDA, J. (2000): "Les ceràmiques arcaiques de la Palaià Polis d'Empòrion", Monografies Emporitanes, 11, 285-346.

BELÉN, M., PEREIRA, J. (1985): “Cerámicas a torno con decoración pintada en Andalucía", Huelva Arqueológica VII, 307-360.

BENSON, J.L. (1964): "Corinthian Vases at Wellesley College", AJA 68.2, 167-172.

BENSON, J.L. (1981): "Corinthian Vases at Rouen", AJA 85.2, 169-173.

BISI, A.M. (1970): La cerámica púnica. Aspetti e problemi, Napoli.

BOARDMAN, J., Hayes, J. (1973): Excavations at Tocra, 1963-1965, The Archaic Deposits II and
Later Deposits, The British School of Archaeology at Athens, The Society for Libyan studies.

BRONEER, O. (1955): "Excavations at Isthmia", Hesperia XXIV.2, 110-141.

CABRERA, P. (1988-1989): "El comercio foceo en Huelva: cronología y fisionomía", Tartessos y Huelva, Huelva Arqueológica X-XI.3, 43-100.

CABRERA, P. (1996): "Emporion y el comercio griego arcaico en el Nordeste de la Península Ibérica", Formes archaïques et arts ibériques, Collection de la Casa de Velázquez 59, 43-54.

CABRERA, P. (2000): "El comercio jonio arcaico en la Península Ibérica”, P. Cabrera y M.Santos (Coords.): Ceràmiques jònies d'època arcaica: centres de producció i comercialització al mediterrani occidental, 165-175.

CASAS, J., SOLER, V. (2000): "Materials arcaics del jaciment de mas Gusó (Bellcaire d'Empordà)", Monografies emporitanes, 11, 347-360.

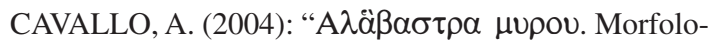
gia, uso e diffusione degli alabastra in alabastro calcareo nel mondo greco e greco-coloniale tra VI e IV secolo a.C.: i rinvenimenti di Locri Epizefiri", $R S L$ LXX, 235-274.

CILIA, E. (1980-1981): "Cozzo Matrice - Scavi 1979", Atti del V congresso internazionale di studi sulla Sicilia antica, Kokalos, XXVI-XXVII.2, 613-619.

DeCAROLIS, E. (1991): Ceramica Corinzia, Gruppo Archeologico Romano, 38, Roma.

DEPPERT, K (1964): Corpus Vasorum Antiquorum, Deutschland, Frankfurt am Main, Band 1, CVA Band 25, München.

ESCACENA, J.L., IZQUIERDO, R. (1996): "Caura protohistórica”, Revista de Arqueología 184, 16-25. 
FERNÁNDEZ, J.H., MEZQUIDA, A. (1997): "Recipientes prerromanos", dins "Vídrios del Puig des Molins (Eivissa), la colección de D. José Costa Picarol", Treballs del Museu Arqueològic d'Eivissa $i$ Formentera 37, 41-56.

GÓMEZ-BELLARD, C. (1990): La colonización fenicia de la isla de Ibiza, EAE 157, Madrid.

GÓMEZ-BELLARD, C. (2000): "La cerámica fenicia de Ibiza", in P.Bartoloni y L.Campanella (Eds.): $L a$ ceramica fenicia di Sardegna. Dati, problematiche, confronti, Atti del I congresso Internazionale Sulcitano, 175-191.

GONZÁLEZ-PRATS, A. (1979a): Excavaciones en el yacimiento protohistórico de La Peña Negra, Crevillente (Alicante), ( $1^{a}$ y $2^{a}$ campañas), EAE 99, Madrid.

GONZÁLEZ-PRATS, A. (1999): "Las cerámicas fenicias de la provincia de Alicante", in A.GonzálezPrats (Ed.): La cerámica fenicia en Occidente: centros de producción y áreas de comercio, Actas del I Seminario Internacional sobre Temas Fenicios, (Guardamar del Segura 1997), 111-128.

GONZÁLEZ-PRATS, A., RUIZ, E., GARCIA, A. (1999): "La Fonteta, 1997”, A.González-Prats (Ed.): La cerámica fenicia en Occidente: centros de producción y áreas de comercio, Actas del I Seminario Internacional sobre Temas Fenicios, (Guardamar del Segura 1997), 257-301.

GRAN-AYMERICH, J.M. (1988): “Cerámicas griegas y etruscas de Málaga. Excavaciones de 1980 a 1986", AEspA 61, 201-221

HOPPER, R.J. (1949): "Addenda to Necrocorinthia", BSA, XLV, 1 i ss.

JIMÉNEZ, J., ORTEGA, J. (2004) : La cerámica griega en Extremadura, Cuadernos Emeritenses, 28, Mérida.

KUNZE-GÖTTE, E. (1965): Corpus Vasorum Antiquorum, Deutschland, Stuttgart, Württembergisches Landesmuseum, Band 1, CVA Band 26, München.

LAWRENCE, P. (1959): "The Corinthian Chimera Painter", AJA 63.4, 349-363.

LAWRENCE, P. (1964): "Five Grave Groups from the Corinthia", Hesperia 33.2, 89-107.

LULLIES, R. (1972): Corpus Vasorum Antiquorum, Deutschland, Kassel, Antikenabteilung der staatlichen Kunstsammlungen, Band 1, CVA Band 35, München.

MALUQUER, J. (1984a): La necrópolis de la Loma de Peinado, Casillas de Martos (Jaén), PIP VI, Universitat de Barcelona, Barcelona.

MALUQUER, J. (1984b): La necrópolis paleoibérica de "Mas de Mussols", Tortosa (Tarragona), PIP VIII, Universitat de Barcelona, Barcelona.

NEWHALL, A., BENSON, J.L. (1984): CORINTH XV.III, The potter's quarter, The pottery. American School of Classical studies at Athens, Princetown, New Jersey.
OLMOS, R. (1985): "Nuevos enfoques para el estudio de la cerámica y de los bronces griegos de España: una primera aproximación al problema de la helenización”, M.Picazo i E.Sanmartí (orgś): Cerámiques gregues $i$ helenístiques a la Península Ibèrica, Empúries 18-20 març de 1983, Barcelona, 7-17.

PAYNE, H. (1931): Necrocorinthia, a study of Corinthian art in the archaic period, Oxford and the Clarendon Press.

RAMÓN, E. (1989): "Un arybalos procedent de la necròpolis de Milmanda (Vimbodí)", Aplec de Treballs del Centre d'Estudis de la Conca de Barberà, 9, 123-134.

RAMÓN, E. (1995): "La necròpolis protohistòrica de Milmanda (Vimbodí)”, Citerior, I, 107-117, 1995.

RAMÓN, J. (1982): “Cuestiones de comercio arcaico: "frascos de aceite perfumado en el Mediterráneo central y occidental", Ampurias 44, 17-41.

RAMÓN, J. (1999): "La cerámica fenicia a torno de sa Caleta (Eivissa)", in A.González-Prats (Ed.): $L a$ cerámica fenicia en Occidente: centros de producción y áreas de comercio, Actas del I Seminario Internacional sobre Temas Fenicios, (Guardamar del Segura 1997), 149-214.

ROEBUCK, M.C., ROEBUCK, C. (1955): "A prize aryballos", Hesperia XXIV.2, 158-163.

ROUILLARD, P. (1977): "Fragmentos griegos de estilo geométrico y corintio medio en Huelva", Huelva arqueológica III, 397-401.

SHEFTON, B.B. (1982): "Greeks and Greek Imports in the South of the Iberian Peninsula. The archaeological evidence", H.G.Niemeyer (dir.): Phönizier im Westen, MB 8, 337-370.

TORRES, M. (1999): Sociedad y mundo funerario en Tartessos, BAH, 3, Madrid.

TRIAS de ARRIBAS, G. (1967): Cerámicas griegas de la Península Ibérica, Publicaciones de Arqueología hispánica, II, Serie primera, Monografías sobre cerámicas hispánicas, 2, 2 vols., València.

URE, P.N. (1910): "Excavations at Rithsona in Boeotia", JHS 30, 336-356.

URE, P.N. (1934): Aryballoi \& Figurines from Rhitsona in Boeotia, Cambridge University Press.

URE, P.N. (1946): "Ring Aryballoi”, Hesperia 15.1, 3850.

VALENZA-MELE, N. (1991): "Vita dell'aldilá e corredi funerari: Evoluzione comparate", DHA 17.2, 149-174.

VIVES, J. (2005): Negociando encuentros. Situaciones coloniales e intercambios en la costa oriental de la Península Ibérica (ss. VIII-VI a.C.), Cuadernos de Arqueología Mediterránea 12, 2005, Barcelona.

Recibido el 26-04-06 Aceptado el 13-06-06 